\title{
Time between skin incision and delivery during cesarean
}

\author{
Jana N. Rossouw \& David Hall \\ Department of Obstetrics and Gynecology, Tygerberg Hospital, Stellenbosch University, \\ Cape Town, South Africa \\ Justin Harvey \\ Centre for Statistical Consultation, Stellenbosch University, Cape Town, South Africa
}

\section{$\underline{\text { Abstract }}$ \\ Objective}

To investigate factors influencing skin incision-to-delivery time (including sub-divisions thereof) and the effect of these surgical intervals on immediate neonatal outcome.

\section{Methods}

A prospective cohort analysis was conducted of all women undergoing cesarean delivery at Tygerberg Hospital, Cape Town, South Africa, from May 24 to November 2, 2010. Three surgical intervals were evaluated: skin incision to myometrium, myometrium to delivery, and skin incision to delivery. Neonatal outcome was assessed by the 5-minute Apgar score.

\section{Results}

Of 1120 cesarean deliveries recorded during the study period, $77.2 \%$ were emergency procedures, which were performed more quickly at all surgical planes $(P<0.01)$. Adhesions in the surgical field were present in $7.4 \%$ of all primary procedures versus $67.7 \%$ of all third procedures $(P<0.001)$. The skin incision-to-delivery time was significantly extended among repeat procedures $(P<0.001)$ and increased progressively with degree of obesity $(P<0.001)$. Although the 3 surgical intervals were calculated individually, none of the median values correlated with a 5-minute Apgar score below 7 for emergency deliveries.

\section{Conclusion}

Repeat procedures, adhesions, and obesity prolonged the time taken for cesarean delivery. Nevertheless, the effect of these factors on the 5-minute Apgar score was minimal.

\section{Keywords}

Adhesions; Apgar score; Cesarean delivery; Incision-to-delivery time; Obesity

\section{Introduction}

Cesarean delivery is the most frequent major surgical intervention in obstetrics. As this procedure can be performed at various levels of healthcare, most health systems categorize patients requiring cesarean delivery into 3 levels of care. The goal of such categorization is to provide appropriate surgical expertise for patients where operative complications are 
anticipated [1]. The rising rate of cesarean delivery observed in many countries is a great cause for concern [2] and [3]. Recurrent use of cesarean delivery is associated with increased intra-abdominal adhesions. Furthermore, placenta previa and morbidly adherent placentas increase the risk of intraoperative complications, such as the need for blood transfusion, bladder injury [4], cesarean hysterectomy, and death. A study performed in the Netherlands showed that placenta accreta was the most frequent indication for peripartum hysterectomy and was significantly associated with cesarean delivery in the current or previous pregnancy [5].

Previous cesarean deliveries increase the risk of adhesions in the operative field and prolong the delivery time [2] and [6]. Some studies have demonstrated that incision-to-delivery times (particularly the myometrium-to-delivery time) influence the Apgar scores of the newborn [7] and [8]. This finding supports the opinion that slow delivery may be to the detriment of the neonate; however, these data have not been confirmed in other studies [9].

Uncertainty clearly exists concerning the importance of the incision-to-delivery time. The aim of the present study was, therefore, to investigate factors influencing the skin incisionto-delivery time (and sub-divisions thereof) and to determine the influence of these surgical intervals on immediate neonatal outcome.

\section{Materials and methods}

A prospective cohort analysis was conducted of all women who underwent elective or emergency cesarean delivery at Tygerberg Hospital, Cape Town, South Africa, from May 24 to November 2, 2010. The study center was a secondary and tertiary referral unit. Elective cesarean deliveries were defined as those performed before the onset of labor. For the purposes of the present study, surgeries performed for failed induction of labor were classified as emergency cesarean deliveries. The Health Research Ethics Committee of Stellenbosch University, Cape Town, South Africa, approved the study design (reference $\mathrm{N}$ $10 / 02 / 32$ ). Owing to the fact that the present study was conducted in the form of a prospective audit, waiver of consent was approved by the Health Research Ethics Committee.

Data were collected prospectively by means of a datasheet that was continuously available in the operating theaters. Before and during the present study, the surgeons, theater sisters, and nurses were informed of the importance of precise time measurements. In practice, the timings were called out to the theater nurse as the operation proceeded; only completed minutes were measured. Immediately after the procedure, other information required by the study investigators was completed by the surgeon. All datasheets were checked by the principal investigator and, where possible, any remaining data added. Data collection was anonymous and only the principal investigator had access to the log connecting a patient's study number to her medical file number.

The main outcome measures were surgical interval and factors that might influence these intervals. Surgical intervals were measured as follows: skin incision to myometrium (S-M), myometrium to delivery (M-D), and skin incision to delivery (S-D). In the case of multiple 
pregnancies, the S-D time of the first newborn was used for the analysis. Obesity was defined as a body mass index (BMI, calculated as weight in kilograms divided by the square of height in meters) of 30 or more at the time of booking of the current pregnancy. Immediate neonatal outcome was defined on the basis of the 5-minute Apgar score.

The data were captured in an Excel (Microsoft, Redmond, WA, USA) spreadsheet and analyzed using STATISTICA version 9.0 (StatSoft, Tulsa, OK, USA). Data were expressed as number (percentage) or median (range). Differences in means were analyzed using the 2tailed Student $t$ test; the Mann-Whitney $U$ test was used for data not normally distributed. The $\chi^{2}$ test was applied to qualitative variables with the Fisher method (for $2 \times 2$ contingency tables) when the expected frequency was below 5. A 1-way analysis of variance with a post hoc Bonferroni adjustment was performed for comparisons of more than 2 groups. A Kruskal-Wallis test was performed where data were not normally distributed. A $P$ value below 0.05 was considered statistically significant.

\section{Results}

During the study period, 4009 deliveries were performed (birth weight $>500 \mathrm{~g}$ ). The total number of cesarean deliveries was 1123 (28.0\%). Of the 1120 cesarean deliveries with data available, $22.8 \%$ were elective and $77.2 \%$ were emergency procedures. The level of surgical experience was as follows: medical officers (54.1\%), registrars (residents/trainee specialists; $25.4 \%)$, specialists (15.0\%), interns (under supervision; $3.5 \%)$, and community service doctors $(2.0 \%)$. The clinical characteristics of the study group at the time of surgery are shown in Table 1.

The details of the 391 women (35.2\%) who had undergone any previous abdominal surgery are shown in Table 2. With regard to the type of skin incision used during cesarean delivery, $88.4 \%$ of 1110 women underwent a Pfannenstiel incision, $7.3 \%$ a midline vertical incision, and $4.3 \%$ a Joel-Cohen incision. At the uterine level, almost all of the incisions were transverse in the lower segment (97.4\%), whereas $1.2 \%$ were classic uterine incisions and $1.4 \%$ were other incisions. Data regarding surgical intervals and factors potentially influencing delivery time are shown in $\underline{\text { Table } 3}$.

Most cesarean deliveries were performed by medical officers or registrars. The median S-D time was 6 minutes (range, <1-47) for medical officers and 5 minutes (range, 1-28) for registrars $(P<0.001)$. The presence of adhesions in the operative area can influence surgical times [1] and [5]. For this reason, intraoperative findings of adhesions were carefully noted (Table 4).

Ease of entry into the abdomen was compared across the 5 different levels of surgical experience. A $\chi^{2}$ test found no significant association between the expressed ease of entry into the abdomen and surgical experience $(P=0.32)$. The ease of entry for the 3 largest experience groups (medical officers, registrars, and specialists) was also evaluated. No significant association was evident between these groups either $(P=0.55)$. Major intraoperative complications were rarely linked to adhesions in the present study. Cesarean hysterectomies ( $n=5)$ were performed for other reasons, such as morbidly adherent placenta or uterine atony. No intraoperative bladder injuries were reported. 
Immediate neonatal outcome was assessed on the basis of a 5-minute Apgar score below 7 (Table 5). This criterion was recorded in 47 (5.6\%) of the 840 emergency cesarean deliveries and in $7(2.8 \%)$ of the 250 elective cesarean deliveries $(P=0.07)$.

\section{Discussion}

Factors leading to prolonged incision-to-delivery time may influence neonatal outcomes after cesarean delivery and should be taken into account when choosing the level of care. When compared with elective surgeries, emergency procedures were performed more quickly at all surgical planes $(P<0.01)$. Adhesions in the surgical field were present in $7.4 \%$ of all primary and $67.7 \%$ of all third procedures $(P<0.001)$. The skin incision-to-delivery time was significantly extended among repeat procedures $(P<0.001)$ and increased progressively with degree of obesity $(P<0.001)$. The type of skin incision also influenced the total delivery time with Joel-Cohen incision providing the fastest time, followed by Pfannenstiel incision, and midline vertical incision $(P=0.005)$, which is in agreement with the findings of Stark and Finkel [10]. Although S-M, M-D, and S-D times were calculated individually, none of the median values correlated with 5-minute Apgar scores below 7 for emergency deliveries.

Obesity was previously considered a problem of high-income countries but has now also become an issue for low-income countries [11]. The large-scale National Health and Nutrition Examination Survey conducted in the USA found that $35.5 \%$ of women aged 2039 years were obese $(\mathrm{BMI} \geq 30)$ when adjusted for age [12]. In South Africa, $44 \%$ of pregnant women were reported to have a BMI of at least 30 [13]. Women with obesity present many challenges both during normal labor and when cesarean delivery becomes necessary. For this reason, all pregnant women with morbid obesity (BMI > 45) who attend Tygerberg Hospital are evaluated by an obstetric anesthetist close to term [14]. In the present study, half of all women undergoing cesarean delivery were obese and approximately $20.0 \%$ were morbidly obese. The S-D times were significantly extended $(P<0.001)$ among obese women $(\mathrm{BMI} \geq 30)$, mainly reflecting prolonged S-M times. Few studies have investigated the influence of obesity on delivery times. However, the findings of the present study differ from those of Tulandi et al. [2], who found no correlation between $\mathrm{BMI}$ and delivery time in a leaner population (BMI $\leq 34.0)$, while a study performed in Saudi Arabia found no correlation between BMI and bladder injury [4].

In a retrospective cohort of first repeat cesarean deliveries performed at a teaching institution, Morales et al. [15] found no correlation between level of surgical training among registrars and S-D time. In the present study, several levels of surgical expertise were recorded. Approximately $80.0 \%$ of the cesarean deliveries were performed by medical officers and registrars and although differences in the S-D times were statistically significant $(P<0.001)$ such differences may not be clinically significant. As expected, the present study showed that abdominal adhesions increased with the number of previous cesarean deliveries $(P<0.001)$ making surgical access more difficult $(P<0.001)$. These findings confirm the results of other studies that have shown the delivery time of repeat cesarean deliveries to be markedly prolonged among women with adhesions [2] and [6]. This information should be considered when deciding the level of care in cases where the anatomy may be disturbed by probable adhesions [16]. In the Saudi Arabian study [3], 95\% 
of cesarean deliveries were performed by senior residents or specialists. In the present study, the ease of surgical entry into the abdomen reported by the different levels of surgeon did not differ. Although it may be assumed that specialists were involved in cases with the highest anticipated risk, it would be difficult to preempt all cases owing to the partially unpredictable nature of adhesions.

An Israeli study published in 1994 found that 5-minute Apgar scores of 7 or less were significantly related to multiple cesarean deliveries even after controlling for the effect of gestational age [17]. Gestational age was not taken into account in the present study. However, an important contribution regarding surgical delivery intervals was made by Maayan-Metzger et al. [9] in 2010. This large study, which involved only elective cesarean deliveries under regional anesthesia, investigated skin incision-to-delivery and uterine incision-to-delivery times and assessed their influence on the short-term outcomes of singleton, full-term infants. The authors reported that the duration of these intervals had no significant impact on the measured neonatal parameters, including 5-minute Apgar score less than 7, and concluded that there is a relatively large safety margin for obstetricians from the time of the skin and uterine incisions [9]. The present study included all cesarean deliveries performed at a single center over a defined time period. As the number of elective deliveries with low 5-minute Apgar scores was small $(n=7)$, only emergency procedures were used to evaluate the relationship between surgical interval and Apgar score. A wider range of surgical delivery intervals was tested than in the study of MaayanMetzger et al. [9]; however, none of the median values correlated with 5-minute Apgar scores below 7 for the emergency deliveries.

Factors such as adhesions, obesity, or surgical experience may influence cesarean delivery times; however, the circumstances influencing the immediate neonatal outcome are also multifactorial. The need to deliver swiftly becomes more urgent in certain emergency procedures (e.g. in the presence of pathologic findings by cardiotocography). Although the present study included all cesarean deliveries, the working definition of emergency cesarean delivery was too broad to investigate the influence of the underlying maternal and/or fetal condition (e.g. pre-eclampsia or growth restriction) on the Apgar score. Given the general findings on the surgical delivery intervals, the maternal and fetal factors in specific cases may be the more important determinants of outcome. In addition, evaluation of umbilical cord blood gases may have been considered as a marker of immediate neonatal outcome but this approach is not standard practice for all cesarean deliveries preformed at the study center. Finally, the type of anesthetic used may be another factor determining outcome [9] but this issue was not investigated in the present study.

In conclusion, the present study showed that surgical considerations, such as obesity and adhesions, require an appropriate level of skill during repeat cesarean deliveries. The lack of association between S-D times and the 5-minute Apgar score provide general reassurance and surgeons should not sacrifice careful surgical technique for hasty delivery of the neonate.

Conflict of interest

The authors have no conflicts of interest. 


\section{5. $\underline{\text { Tables }}$}

Table 1.

Clinical characteristics of the study group at the time of surgery $(n=1123) \cdot \underline{a}$

\begin{tabular}{||l|l||}
\hline \multicolumn{1}{|c|}{ Characteristic } & Distribution \\
\hline \hline Age, $y$ & $28(12-48)$ \\
\hline Parity & $1(0-6)$ \\
\hline Primigravida $\underline{b}$ & $346(30.0)$ \\
\hline Any previous abdominal surgery $\underline{c}$ & $391(35.4)$ \\
\hline \hline Previous cesarean delivery $\underline{\mathrm{d}}$ & $366(33.2)$ \\
\hline BMI $\underline{\mathrm{e}}$ & \\
\hline$<30$ & $469(50.1)$ \\
\hline \hline $30.0-39.9$ & $289(30.8)$ \\
\hline$\geq 40$ & $179(19.1)$ \\
\hline
\end{tabular}

Abbreviation: BMI, body mass index (calculated as weight in kilograms divided by the square of height in meters).

a Values are given as median (range) or number (percentage).

b Gravidity data available for 1116 women.

c Surgical data available for 1105 women.

d Surgical data available for 1105 women.

e BMI data available for 937 women. 
Table 2.

Evaluation of previous abdominal surgery $(n=1110)$.

\begin{tabular}{|l|l|}
\hline Procedure or indication & Distribution \\
\hline \hline General & \\
\hline Lower abdominal surgery & $386(34.8)$ \\
\hline Upper abdominal surgery & $10(0.9)$ \\
\hline Specific & \\
\hline No. of cesarean deliveries & \\
\hline \hline 1 & $210(59.3)$ \\
\hline \hline 2 & $134(37.9)$ \\
\hline \hline 3 & $10(2.8)$ \\
\hline \hline$>3$ & $0(0.0)$ \\
\hline Ectopic pregnancy & $18(4.6)$ \\
\hline \hline Appendectomy & $9(2.3)$ \\
\hline \hline Laparoscopy & $7(1.8)$ \\
\hline \hline
\end{tabular}

a Values are given as number (percentage).

b Some patients had previous upper and lower abdominal surgery. 
Table 3.

Evaluation of surgical interval. ${ }^{\text {and }}$

\begin{tabular}{|c|c|c|c|c|c|c|}
\hline \multirow[t]{2}{*}{ Characteristic } & \multicolumn{2}{|c|}{$\begin{array}{c}\text { Skin to } \\
\text { myometrium }\end{array}$} & \multicolumn{2}{|c|}{$\begin{array}{c}\text { Myometrium to } \\
\text { delivery }\end{array}$} & \multicolumn{2}{|c|}{ Skin to delivery } \\
\hline & Time, $\min$ & $P$ value & Time, $\min$ & $P$ value & Time, $\min$ & $P$ value \\
\hline $\begin{array}{l}\text { Cesarean } \\
\text { delivery }\end{array}$ & & $<0.001$ & & $\mid<0.001$ & & $\mid<0.001$ \\
\hline Primary & $3(<1-18)$ & & $1(<1-15)$ & & $5(<1-26)$ & \\
\hline Repeat & $6(1-45)$ & & $2(<1-28)$ & & $8(1-47)$ & \\
\hline Obesity (BMI) & & $<0.001$ & & $<0.001$ & & $<0.001$ \\
\hline$<25$ & $3(<1-18) \subseteq$ & & $1(<1-15) \subseteq$ & & $5(1-21) \subseteq$ & \\
\hline $25.01-29.99$ & $3(<1-35) \subseteq$ & & $1(<1-11) \subseteq$ & & $5(1-36) \mathrm{c}$ & \\
\hline $30.00-39.99$ & $4(1-33) \underline{d}$ & & $2(<1-9) \subseteq$ & & $6(1-39) \underline{d}$ & \\
\hline $40.00-49.99$ & $5(1-45) \underline{d}$ & & $2(<1-12) \frac{c, d}{2}$ & & $7(2-47) \underline{d}$ & \\
\hline$>50$ & $5(1-35) \stackrel{d}{d}$ & & $2(1-15) \subseteq$ & & $8(3-37) \underline{d}$ & \\
\hline Skin incision & & $<0.001$ & & 0.16 & & 0.005 \\
\hline Joel-Cohen & 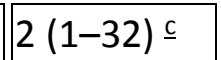 & & $1(1-4) \div$ & & 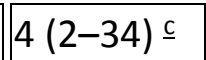 & \\
\hline Pfannenstiel & $\mid 4(0-45) \stackrel{c}{-}$ & & $1(<1-28) \subseteq$ & & $6(<1-47)$ & \\
\hline Midline vertical & $5(1-26) \stackrel{d}{d}$ & & $1(<1-7) \subseteq$ & & $6(2-29) \underline{d}$ & \\
\hline Indication & & $<0.001$ & & 0.002 & & $<0.001$ \\
\hline Elective & $6(1-35)$ & & $2(<1-28)$ & & $8(2-39)$ & \\
\hline Emergency & $3(<1-45)$ & & $1(<1-13)$ & & $5(<1-47)$ & \\
\hline
\end{tabular}

Abbreviation: BMI, body mass index (calculated as weight in kilograms divided by the square of height in meters).

a Values are given as median (range) unless otherwise indicated.

b Patients with available data only.

c,d Superscripts indicate the results of post hoc pairwise comparisons. Categories containing a superscript ${ }^{c}$ are significantly different only from those not containing a superscript ${ }^{c}$.

Categories with a superscript ${ }^{d}$ are significantly different only from those not containing a superscript ${ }^{d}$. 
References

1. K. Cullinan Health services in South Africa: A basic introduction www.health-e.org.za [Published January 2006]

2. T. Tulandi, M. Agdi, A. Zarei, L. Miner, V. Sikirica Adhesion development and morbidity after repeat cesarean delivery Am J Obstet Gynecol, 201 (1) (2009), pp. 56.e1-56.e6

3. J.A. Martin, B.E. Hamilton, P.D. Sutton, S.J. Ventura, F. Menacker, S. Kirmeyer et al. Births: Final data for 2006 Natl Vital Stat Rep, 57 (7) (2009), pp. 1-102

4. M.S. Rahman, T. Gasem, S.A. Al Suleiman, F.E. Al Jama, S. Burshaid, J. Rahman Bladder injuries during cesarean section in a University Hospital: a 25-year review Arch Gynecol Obstet, 279 (3) (2009), pp. 349-352

5. Kwee, M.L. Bots, G.H. Visser, H.W. Bruinse Emergency peripartum hysterectomy: A prospective study in The Netherlands Eur J Obstet Gynecol Reprod Biol, 124 (2) (2006), pp. 187-192

6. M.B. Greenberg, K. Daniels, Y.J. Blumenfeld, A.B. Caughey, D.J. Lyell Do adhesions at repeat cesarean delay delivery of the newborn? Am J Obstet Gynecol, 205 (4) (2011), pp. 380.e1-380.e5

7. G.B. Theron, J.T. Nel, H.J. Odendaal Fetal heart monitoring during general anaesthesia for caesarean section S Afr Med J (“Geneeskunde”), 68 (10) (1985), pp. 317-327

8. S.K. Kamat, M.V. Shah, L.S. Chaudhary, S. Pandya, M.M. Bhatt Effect of inductiondelivery and uterine-delivery on apgar scoring of the newborn J Postgrad Med, 37 (3) (1991), pp. 125-127

9. Maayan-Metzger, I. Schushan-Eisen, L. Todris, A. Etchin, J. Kuint The effect of time intervals on neonatal outcome in elective cesarean delivery at term under regional anesthesia Int J Gynecol Obstet, 111 (3) (2010), pp. 224-228

10. M. Stark, A.R. Finkel Comparison between the Joel-Cohen and Pfannenstiel incisions in cesarean section Eur J Obstet Gynecol Reprod Biol, 53 (2) (1994), pp. 121-122

11. R.T. Erasmus, D.J. Soita, M.S. Hassan, E. Blanco-Blanco, Z. Vergotine, A.P. Kengne et al. High prevalence of diabetes mellitus and metabolic syndrome in a South African coloured population: Baseline data of a study in Bellville, Cape Town S Afr Med J, 102 (11) (2012), pp. 841-844

12. K.M. Flegal, M.D. Carroll, C.L. Ogden, L.R. Curtin Prevalence and trends in obesity among US adults, 1999-2008 JAMA, 303 (3) (2010), pp. 235-241

13. J.K. Basu, C.M. Jeketera, D. Basu Obesity and its outcomes among pregnant South African women Int J Gynecol Obstet, 110 (2) (2010), pp. 101-104

14. E. Ngoga, D. Hall, F. Mattheyse, D. Grove Outcome of pregnancy in the morbidly obese women S Afr Fam Pract, 51 (1) (2009), pp. 39-41

15. K.J. Morales, M.C. Gordon, G.W. Bates Jr. Postcesarean delivery adhesions associated with delayed delivery of infant Am J Obstet Gynecol, 196 (5) (2007), pp. 461.e1-461.e6

16. J. Mowat, J. Bonnar Abdominal wound dehiscence after caesarean section $\mathrm{Br}$ Med J, 2 (5756) (1971), pp. 256-257

17. D.S. Seidman, I. Paz, A. Nadu, S. Dollberg, D.K. Stevenson, R. Gale et al. Are multiple cesarean sections safe? Eur J Obstet Gynecol Reprod Biol, 57 (1) (1994), pp. 7-12 
